EPJ Web of Conferences 101, 06055 (2015)

DOI: $10.1051 /$ epjconf/ 201510106055

(C) Owned by the authors, published by EDP Sciences, 2015

\title{
Empirical solar/stellar cycle simulations
}

\author{
Ângela R. G. Santos ${ }^{1,2, a}$, Margarida S. Cunha ${ }^{1}$, and Pedro P. Avelino ${ }^{1,2}$ \\ 1 Instituto de Astrofísica e Ciências do Espaço, Universidade do Porto, CAUP, Rua das Estrelas, 4150- \\ 762 Porto, Portugal \\ 2 Departamento de Física e Astronomia, Faculdade de Ciências, Universidade do Porto, Rua do \\ Campo Alegre 687, 4169-007 Porto, Portugal
}

\begin{abstract}
As a result of the magnetic cycle, the properties of the solar oscillations vary periodically. With the recent discovery of manifestations of activity cycles in the seismic data of other stars, the understanding of the different contributions to such variations becomes even more important. With this in mind, we built an empirical parameterised model able to reproduce the properties of the sunspot cycle. The resulting simulations can be used to estimate the magnetic-induced frequency shifts.
\end{abstract}

\section{Introduction}

For many years, the activity-related variations in the oscillation properties of the solar acoustic modes have been studied [1, e.g] and, more recently, such variations were detected in a solar-like CoRoT star [2]. However, the different processes contributing to those variations are not completely understood. We develop an empirical parameterised model able to reproduce the sunspot cycle. Among other applications, these simulations can be used to estimate the activity-related frequency shifts and thus the direct effect of spots on the oscillations.

\section{Empirical sunspot cycle model and the spot-induced frequency shifts}

In order to obtain reasonable simulations of the sunspot cycle, we base our model on observational constraints, such as the number of sunspot groups, the total area covered by spots, the group lifetimes, and their latitudinal distribution. At each time step, a given number of groups are formed and distributed in longitude and latitude. The latitudinal distribution of the generated groups considers the equatorward drift and the varying width of the sunspot formation zone over the cycle. Each group is assumed to follow the rotation of the solar surface according to its latitude and only groups in the visible side of the disc are considered. The maximum area of each group is obtained from the area distribution observed in the Sun. The group's lifetime is obtained from a modified version of the Gnevyshev-Waldmeier rule which accounts for a deviation from the linear relation between the maximum area and the lifetime for the smaller spots. The time evolution of the area of individual groups is also accounted for. Fig. 1 shows the results of a simulation obtained with our empirical model. The apparent agreement between real and simulated data is clear and was also verified with the Kolmogorov-Smirnov test. Based on the variational principle [3], we derived a relation for the frequency shifts induced by the spots at each time (eq. 1). The relative frequency shifts

$$
\frac{\delta v(t)}{v} \propto-\Delta \delta_{c h} \sum_{i=1}^{N(t)}\left[\left(P_{l}^{m}\left(\cos \theta_{i}\right)\right)^{2} A_{i}\right],
$$

\footnotetext{
a e-mail: asantos@astro.up.pt
}

This is an Open Access article distributed under the terms of the Creative Commons Attribution License 4.0, which permits unrestricted use, distribution, and reproduction in any medium, provided the original work is properly cited. 

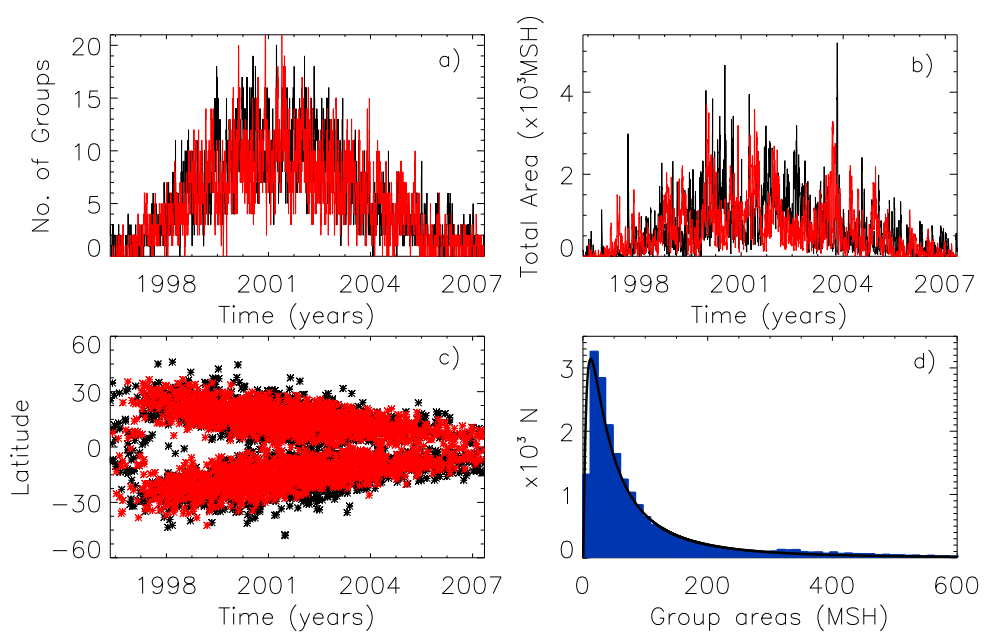

Fig. 1. Comparison between simulated (red) and real data (black) for: a) number of sunspot groups, b) total area covered by sunspot groups, c) group latitudes, d) accumulated area distribution where the solid line represents the observed distribution.

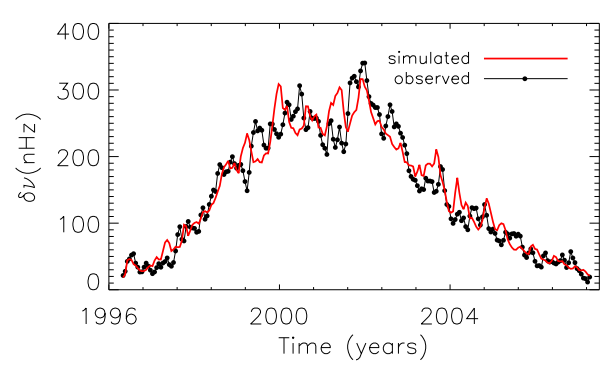

Fig. 2. Observed frequency shifts (black dots with the solid line to guide the eye; [4]) and the resulting combination of a smooth component and the spot-induced frequency shifts computed from our simulations (red solid line). The spotinduced component contributes $\sim 20 \%$ to the total frequency shifts.

depend on the phase shift induced by the spots on the acoustic wave (here we assume a fixed characteristic value, $\Delta \delta_{c h}$ ), on the group areas, $A_{i}$, and colatitudes, $\theta_{i}$, and on the angular degree, $l$, and azimuthal order, $m$, of the mode. Applying this relation both to simulations and to real sunspot data, we find that the observed frequency shifts must result from a combination of a smooth (possibly associated to global solar magnetic field) and a spot-induced component (Fig. 2).

\section{Conclusions}

Our empirical model is able to reproduce well the properties of the sunspot cycle. Concerning other solar-like pulsators for which less information is available, we intend to apply a simplified version of the model and then estimate the frequency shifts that may be expected for those stars. Our model has potentially other applications, such as in the study of transit and radial velocity signals for exoplanet search.

We are very grateful to S.C. Tripathy for providing the data points of the observed frequency shifts. We thank to FCT (SFRH/BD/88032/2012, and Investigador FCT contracts), POPH/FSE (EC), and FP7-SPACE- 2012-1 for the financial support.

\section{References}

1. M.F. Woodard, R.W. Noyes, Nature 318, 449 (1985)

2. R.A. García, S. Mathur, J. Ballot, C. Régulo, T.S. Metcalfe, A. Baglin, Science 329, 1032 (2010)

3. M.S. Cunha, D. Gough, Monthly Notices of the Royal Astronomical Society 319, 1020 (2000)

4. S.C. Tripathy, K. Jain, D. Salabert, R.A. García, F. Hill, J.W. Leibacher, Journal of Physics: Conference Series 271, 2055 (2011) 